\title{
Computer Search for Nilpotent Complexes
}

\author{
Robert H. Lewis and Guy D. Moore
}

\section{CONTENTS}

1. Introduction

2. Nilpotent Modules and Spaces

3. Construction of Nilpotent Complexes Via Cellular Chains

4. Computer Representation of Chain Complexes and Homology Modules

5. The Programs

6. An Alternate Approach and Future Work Electronic Availability

References

Partially supported by NSF R.E.U. Grants DMS-8803864, DMS-8900752, and DMS-9100782.
The concept of nilpotency for a topological space is a generalization of simple connectivity. That it is a fruitful generalization was shown by Dror, Kan, Bousfield, Hilton, and others. In 1977 Brown and Kahn proved that the dimension of a nilpotent complex can be read from the ordinary homology groups, just as in the case of a simply connected complex. They also showed that if a nilpotent complex has finite and nontrivial fundamental group, its dimension must be at least 3 .

In 1985 Lewis showed that for any finite nilpotent group there is a (not necessarily finite) three-dimensional nilpotent complex with that fundamental group. The smallest finite nilpotent group for which it was unknown whether a finite threedimensional nilpotent complex exists was $\mathbb{Z}_{2} \oplus \mathbb{Z}_{6}$.

The authors, together with a team of undergraduate students at Fordham University, used computers to search for threedimensional finite nilpotent complexes over groups of the form $\mathbb{Z}_{\mathrm{n}} \oplus \mathbb{Z}_{\mathrm{m}}$. Such complexes were eventually found for $\mathbb{Z}_{2} \oplus \mathbb{Z}_{6}$, $\mathbb{Z}_{2} \oplus \mathbb{Z}_{10}$, and $\mathbb{Z}_{3} \oplus \mathbb{Z}_{6}$.

This article describes the strategy for constructing nilpotent complexes of dimension three, and some of the issues in implementing the computer search. The main computational issues are "normalizing" matrices, especially to the Smith normal form, and mapping matrices over $\mathbb{Z}$ to matrices over $\mathbb{Z}_{\mathrm{p}}$ for various primes $\mathrm{p}$. We conclude with a summary of the complexes discovered and open questions.

\section{INTRODUCTION}

Algebraic topology seeks to translate difficult topological questions into less difficult algebraic ones via functors, such as the homotopy groups $\pi_{*}$ and the homology groups $H_{*}$. The following result is basic [Rotman 1988]:

Theorem 1.1. If $f: X \rightarrow Y$ is a homotopy equivalence, the induced homomorphisms $f_{*}: H_{n} X \rightarrow$ $H_{n} Y$ and $f_{*}: \pi_{n} X \rightarrow \pi_{n} Y$ are isomorphisms for all $n \geq 1$. 
It is natural to seek a converse to this result. From now on we restrict our attention to the category of pointed path connected spaces with the homotopy type of a simplicial complex (or equivalently, of a CW complex). Then [Spanier 1966, pp. 405-406]:

Theorem 1.2 (Whitehead). If $f: X \rightarrow Y$ induces isomorphisms $f_{*}: \pi_{n} X \rightarrow \pi_{n} Y$ for all $n \geq 1$, then $f$ is a homotopy equivalence.

As important as this theorem is, we'd rather deal with the homology groups. They are much more computable because they satisfy the Excision Axiom [Rotman 1988]. For example, if $X$ is an $n^{-}$ dimensional complex, the homology groups $H_{k} X$ vanish for $k>n$. Furthermore, the homology groups reflect the cell structure of the space, in that group presentations for each homology group arise directly from the cell attaching maps. Unfortunately, examples show that Theorem 1.2 fails if $\pi_{n}$ is replaced by $H_{n}$. But if the spaces $X$ and $Y$ are simply connected (whence $H_{1} X=H_{1} Y=0$ ), we do indeed obtain:

Theorem 1.3 (Whitehead). If $X$ and $Y$ are simply connected and $f: X \rightarrow Y$ induces isomorphisms $f_{*}: H_{n} X \rightarrow H_{n} Y$ for all $n \geq 2$, then $f$ is a homotopy equivalence.

Dror [1971] extended Theorem 1.3 to more general spaces called nilpotent complexes, which we will define in the next section. For now, think of a nilpotent space as more general than a simplyconnected space in the same way that a nilpotent group is more general than an abelian group. Indeed, nilpotent CW complexes always have nilpotent fundamental groups.

Theorem 1.4 (Dror). Let $X$ and $Y$ be nilpotent $C W$ complexes. Then $f: X \rightarrow Y$ is a homotopy equivalence if $f$ induces isomorphisms $f_{*}: H_{n} X \rightarrow H_{n} Y$ for all $n \geq 1$.

This result gives us the motivation for producing some simple nilpotent complexes. In particular, one may ask: Given a nilpotent fundamental group $\pi_{1} X$, construct a nilpotent complex of least dimension. Brown and Kahn [1977] showed that if $\pi_{1} X$ is finite and nontrivial, the nilpotent space $X$ must be at least three-dimensional. A natural question is: Can this lower bound be attained? Lewis [1985] gave an affirmative answer and furthermore provided a construction for any finite nilpotent fundamental group. However, the construction only guarantees a finite complex (i.e., a finite number of cells constituting the complex) when $\pi_{1} X$ is a cyclic or a $p$-group. Otherwise a general construction of a finite six-dimensional nilpotent complex was given for any finite nilpotent fundamental group.

Lewis carried out three National Science Foundation R.E.U. (Research Experience for Undergraduates) programs to search for three-dimensional finite nilpotent complexes using computers. Besides coauthor Moore, the undergraduate participants were (in alphabetical order) D. Clark, A. Drisko, B. Farb, A. Gottlieb, J. Martone, A. Mayer, B. Narasimhan, C. Powell, D. Rath, R. Servedio, C. Snyder, P. Socolow, Y. Sussman, E. Wolfe, and E. Zaslow.

A beginning step is to assume $\pi_{1} X \cong \mathbb{Z}_{n} \oplus \mathbb{Z}_{m}$ with $n, m$ 's that make the groups neither cyclic nor $p$-groups. (We exclude cyclic groups and $p$-groups because such groups were already known to admit finite three-dimensional nilpotent complexes.) The smallest such group is $\mathbb{Z}_{2} \oplus \mathbb{Z}_{6}$. The construction has been implemented on Macintosh computers. Programs have been written in Pascal and Fermat, a computer algebra system [Lewis 1996]. The first examples for $\pi_{1} X=\mathbb{Z}_{2} \oplus \mathbb{Z}_{6}$ were found by Moore. Later, examples for $\mathbb{Z}_{2} \oplus \mathbb{Z}_{10}$ and $\mathbb{Z}_{3} \oplus \mathbb{Z}_{6}$ were discovered.

\section{NILPOTENT MODULES AND SPACES}

Recall that a group $G$ is called nilpotent if there exists $G_{k}$ in the lower central series of $G$ such that $G_{k}=\{1\}$. Trivially, abelian groups are nilpotent. It is well known that $p$-groups are nilpotent.

Now let $\pi$ be a group and let $\mathbb{Z} \pi$ the group ring of $\pi$. The ring epimorphism $\varepsilon: \mathbb{Z} \pi \rightarrow \mathbb{Z}$ given by 
$\varepsilon\left(\sum n_{i} x_{i}\right)=\sum n_{i}$ is called the augmentation map. The kernel of $\varepsilon$ is the augmentation ideal, denoted by $I$.

Suppose $M$ is a (left) $\mathbb{Z} \pi$-module. Define submodules of $M$ inductively as follows:

$$
\begin{aligned}
I^{0} M & =M, \\
I^{k} M & =I\left(I^{k-1} M\right)=\left\langle i x: i \in I, x \in I^{k-1} M\right\rangle,
\end{aligned}
$$

for $k \geq 1$. We have the tower of submodules

$$
M=I^{0} M \supset I^{1} M \supset \cdots \supset I^{k-1} M \supset I^{k} M \supset \cdots .
$$

We say that $M$ is $\pi$-trivial if $I M=0$. We say that $M$ is nilpotent if $I^{k} M=0$ for some $k$. Finally, $M$ is perfect if $I M=M$.

If $M$ is a left $\mathbb{Z} \pi$-module, there is a natural "trivializing map" $M \rightarrow M / I M$.

Recall that if $X$ is a pointed topological space there is a natural action of its fundamental group $\pi_{1} X$ on the homology groups of the universal cover $\tilde{X}$. Via this action, the homology groups $H_{n} \tilde{X}$ are modules over the group ring $\mathbb{Z} \pi_{1} X$.

Finally, we have the definition of a nilpotent space:

Definition 2.1. A topological space $X$ is called nilpotent if $\pi=\pi_{1} X$ is a nilpotent group and each $H_{n} \tilde{X}$ is a nilpotent $\mathbb{Z} \pi$-module.

For example, if $X$ is simply connected, $\pi=\pi_{1} X=$ $0, \mathbb{Z} \pi=\mathbb{Z}$ and $I=0$, so $X$ is trivially nilpotent.

We will be concerned with spaces that have only finitely many homology groups, each of which is finitely generated. Such a space is said to be of finite homological type.

For the rest of this paper, $\pi$ always means $\pi_{1} X$ for some space $X$. We often abbreviate " $M$ is a $\mathbb{Z} \pi$-module" to just " $M$ is a $\pi$-module".

\section{CONSTRUCTION OF NILPOTENT COMPLEXES VIA CELLULAR CHAINS}

The homology groups of a CW complex $X$ can be computed with cellular homology [Rotman 1988]. To do this, one defines a chain complex $C_{*} X$ whose group of $n$-chains is the free abelian group on the $n$ cells. Specifically, $C_{k} X=H_{k}\left(X^{k}, X^{k-1}\right.$ ) (singular homology) and $\partial_{k}: C_{k} X \rightarrow C_{k-1} X$ arises from inclusion maps and singular boundary maps.

To construct a nilpotent cell complex $X$ we need not only the cellular chain complex of $X$ but also the $\mathbb{Z} \pi$-module structure on the cellular chain complex of $\tilde{X}$. This means that we attach the cells of $\tilde{X}$ by lifting the attaching map of each cell of $X$. Hence, for each cell of $X$ and each element of $\pi$ there is a corresponding lifted cell of $\tilde{X}$. The result is an equivariant cellular decomposition of $\tilde{X}$, in which each element of $\pi_{1} X$ acts on the cells that make up the universal cover $\tilde{X}$. The chain complex $C_{*} \tilde{X}$ is a complex of free $\pi$-modules and $\pi$-module homomorphism boundary maps. We may imagine the chain complex of $\tilde{X}$ "covering" that of $X$, producing the following commutative diagram, in which each vertical map is induced by the natural map from $\tilde{X} \rightarrow X$ and is a trivializing map:

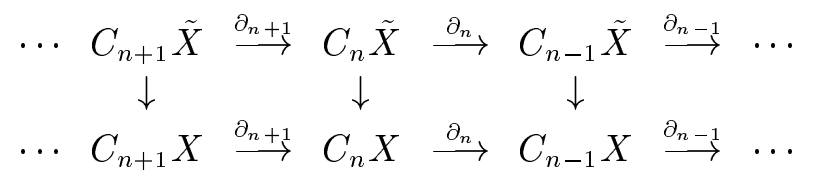

We want to construct chain complexes for $\pi_{1} X=$ $\mathbb{Z}_{n} \oplus \mathbb{Z}_{m}$ such that all the groups and $\pi$-modules are finitely generated abelian groups, the complexes vanish above dimension three, and the homology modules $\mathrm{H}_{2} \tilde{X}$ and $H_{3} \tilde{X}$ are nilpotent (the others being 0).

It is useful to look first at a three-dimensional nilpotent complex for cyclic fundamental group $\pi=$ $\mathbb{Z}_{n}$. Write $\pi$ as the multiplicative group generated by $x$ with $x^{n}=1$, so $\mathbb{Z} \pi \cong \mathbb{Z}[x] /\left\langle x^{n}-1\right\rangle$. Set $T_{x}=x-1, N_{x}=1+x+\cdots+x^{n-1}$. There is a well known free $\mathbb{Z} \pi$ resolution of the $\pi$-trivial module $\mathbb{Z}$ :

$$
\cdots \longrightarrow \mathbb{Z} \pi \stackrel{\partial_{2}}{\longrightarrow} \mathbb{Z} \pi \stackrel{\partial_{1}}{\longrightarrow} \mathbb{Z} \pi \stackrel{\varepsilon}{\longrightarrow} \mathbb{Z} \longrightarrow 0 .
$$

Here $\partial_{i}$ for odd $i$ is multiplication by $T_{x}$ and for even $i$ is multiplication by $N_{x}$. The sequence is exact. There is an Eilenberg-MacLane space $X=$ $K(\pi, 1)$ having one cell in each dimension whose 
universal cover $\tilde{X}$ has exactly the above cellular chain complex. If we simply cut this off at dimension 3 , we get $H_{2} \tilde{X}^{3}=0$ and $H_{3} \tilde{X}^{3}=\mathbb{Z}, \pi$-trivial, so $X^{3}$ is nilpotent.

Now suppose $\pi=\mathbb{Z}_{n} \oplus \mathbb{Z}_{m}$. Again think of $\mathbb{Z}_{n}$ as the group $\left\langle x: x^{n}=1\right\rangle$, and $\mathbb{Z}_{m}$ as $\left\langle y: y^{m}=1\right\rangle$. Then $\mathbb{Z} \pi \cong \mathbb{Z}[x, y] /\left\langle x^{n}-1, y^{m}-1\right\rangle$. As before, we have $N_{x}, N_{y}, T_{x}$, and $T_{y}$. We may obtain $X=K(\pi, 1)$ by taking the product of the spaces $K\left(\mathbb{Z}_{n}, 1\right)$ and $K\left(\mathbb{Z}_{m}, 1\right)$. The chain complex of the universal cover is a free $\mathbb{Z} \pi$ resolution of $\mathbb{Z}$, namely the tensor product of each of the resolutions (3.1) for $n$ and $m$. The sequence ends

$$
\begin{aligned}
\cdots \longrightarrow \mathbb{Z} \pi \oplus \mathbb{Z} \pi \oplus \mathbb{Z} \pi \oplus \mathbb{Z} \pi \stackrel{\partial_{3}}{\longrightarrow} \mathbb{Z} \pi \oplus \mathbb{Z} \pi \oplus \mathbb{Z} \pi \\
\stackrel{\partial_{2}}{\longrightarrow} \mathbb{Z} \pi \oplus \mathbb{Z} \pi \stackrel{\partial_{1}}{\longrightarrow} \mathbb{Z} \pi \stackrel{\varepsilon}{\longrightarrow} \mathbb{Z} \longrightarrow 0 .
\end{aligned}
$$

A little computation shows that $\partial_{1}$ and $\partial_{2}$ are the matrices

$$
\begin{aligned}
\partial_{1} & =\left(\begin{array}{ll}
T_{x} & T_{y}
\end{array}\right), \\
\partial_{2} & =\left(\begin{array}{ccc}
N_{x} & 0 & -T_{y} \\
0 & N_{y} & T_{x}
\end{array}\right) .
\end{aligned}
$$

As in the cyclic case, we may ask whether the three-skeleton $X^{3}$ is nilpotent. This is true if and only if $H_{3} \tilde{X}^{3}$ is a nilpotent $\pi$-module. Rather than compute this module, we may obtain the negative answer using the following result:

Theorem 3.1. Let $\pi$ be a nontrivial finitely generated nilpotent group. Let $X$ be a nilpotent complex of finite homological type with fundamental group $\pi$. Then $\chi(X)=0$, where $\chi(X)$ is the Euler characteristic of $X$. If $\pi$ is finite, $\chi(\tilde{X})=0$.

This result follows from [Lewis 1985, p. 753] and the fact that, for nilpotent complexes, finite homological type is equivalent to the complex being finitely dominated.

Direct calculation reveals that

$$
\begin{aligned}
& \chi\left(\tilde{X}^{3}\right)=-\mathrm{rk}_{\mathbb{Z}} C_{3} \tilde{X}^{3}+\mathrm{rk}_{\mathbb{Z}} C_{2} \tilde{X}^{3} \quad-\mathrm{rk}_{\mathbb{Z}} C_{1} \tilde{X}^{3}+\mathrm{rk}_{\mathbb{Z}} C_{0} \tilde{X}^{3} \\
&=-4 m n+3 m n-2 m n+m n=-2 m n,
\end{aligned}
$$

where $\mathrm{rk}_{\mathbb{Z}}$ denotes the $\mathbb{Z}$-rank. Therefore $X^{3}$ is not nilpotent. Evidently, not only are the three-cells attached incorrectly, there are too many of them. The Euler characteristic decrees that $C_{3} \tilde{X}^{3}$ should be $\mathbb{Z} \pi \oplus \mathbb{Z} \pi$. On the other hand,

$$
\begin{aligned}
& \chi\left(\tilde{X}^{3}\right)= \\
& \quad-\mathrm{rk}_{\mathbb{Z}} H_{3} \tilde{X}^{3}+\mathrm{rk}_{\mathbb{Z}} H_{2} \tilde{X}^{3}-\mathrm{rk}_{\mathbb{Z}} H_{1} \tilde{X}^{3}+\mathrm{rk}_{\mathbb{Z}} H_{0} \tilde{X}^{3} .
\end{aligned}
$$

Therefore $\mathrm{rk}_{\mathbb{Z}} H_{3} \tilde{X}^{3}=\mathrm{rk}_{\mathbb{Z}} H_{2} \tilde{X}^{3}+1$ in a nilpotent $X^{3}$.

Another conceivable way to create a three-dimensional nilpotent complex is to use the iterated join construction for the classifying space of a group, defined in [Milnor 1956]. The four-fold join of $\pi$ modulo the action of $\pi$ is a three-dimensional complex with the right fundamental group. But its Euler characteristic is not zero, so this too is rejected by Theorem 3.1 .

\section{COMPUTER REPRESENTATION OF CHAIN COMPLEXES AND HOMOLOGY MODULES}

We are now ready to describe our basic strategy to construct a three-dimensional nilpotent complex of fundamental group $\pi=\mathbb{Z}_{n} \oplus \mathbb{Z}_{m}$ :

1. Start with the two-skeleton of the $K(\pi, 1)$ constructed in section $3, X^{2}$, with the equivariant chain complex of the universal cover through dimension two. This space has the right fundamental group. Find a way to attach three-cells equivariantly to $\tilde{X}^{2}$, forming $\tilde{X}^{3}$ so that both $H_{3} \tilde{X}^{3}$ and $H_{2} \tilde{X}^{3}$ are nilpotent $\pi$-modules.

2. Represent all maps with integers.

Computer implementation of this strategy requires that

- $\pi$-modules can be represented efficiently;

- equivariant chain complexes can be represented efficiently; and

- homology $\pi$-modules can be computed and recognized as nilpotent.

There is also a more mathematical issue. Assuming that we succeed in constructing an equivariant 
chain complex with the required homology, is there necessarily a topological space (CW complex) realizing that chain complex? Can we "go backwards" from the algebra to the topology? Because the dimension is only three, the answer is yes; see [Lewis 1985].

We now address each of the above three points. The basic approach is to represent everything as integers, rather than as elements of the more abstract rings $\mathbb{Z}_{\pi}$ or $\mathbb{Z}[x, y]$. However, at the end of the paper we will reconsider that part of our strategy.

\section{A. Representation of $\pi$-modules}

First consider free $\pi$-modules. As a $\pi$-module, let us select for $\mathbb{Z}_{\pi}$ the following ordered $\mathbb{Z}$-basis:

$$
\begin{array}{r}
\left\{1, x, \ldots, x^{n-1}, y, x y, \ldots, x^{n-1} y, \ldots, y^{m-1},\right. \\
\left.x y^{m-1}, \ldots, x^{n-1} y^{m-1}\right\} .
\end{array}
$$

An element of the $\pi$-module $\mathbb{Z}_{\pi}$ is therefore a vector of $m n$ integers. As elements of the ring $\mathbb{Z} \pi$, the generators $x$ and $y$ become $m n \times m n$ permutation matrices, which act on the vector on the left.

Next consider $\pi$-modules that are finitely generated abelian groups. Such a group $M$ is isomorphic to a direct sum of cyclic groups. A typical element of $M$ is therefore a vector of, say, $k$ integers. If we keep a record of which components are finite cyclic groups of what order, it is obvious how to add such vectors on the computer. To specify the $\pi$-module structure of $M$, it is sufficient to represent the $x$ and $y$ actions by commuting $k \times k$ matrices, also called $x$ and $y$, such that $x^{n}=1$ and $y^{m}=1$.

\section{B. Representation of Equivariant Chain Complexes}

From previous sections we have the equivariant chain complex of the two-skeleton $\tilde{X}^{2}$ :

$\mathbb{Z} \pi \oplus \mathbb{Z} \pi \oplus \mathbb{Z} \pi \stackrel{\partial_{2}}{\longrightarrow} \mathbb{Z} \pi \oplus \mathbb{Z} \pi \stackrel{\partial_{1}}{\longrightarrow} \mathbb{Z} \pi \stackrel{\varepsilon}{\longrightarrow} \mathbb{Z} \longrightarrow 0$, where

$$
\begin{aligned}
\partial_{1} & =\left(\begin{array}{ll}
T_{x} & T_{y}
\end{array}\right), \\
\partial_{2} & =\left(\begin{array}{ccr}
N_{x} & 0 & -T_{y} \\
0 & N_{y} & T_{x}
\end{array}\right) .
\end{aligned}
$$

But we now represent maps with integers, rather than elements of the group ring. An arbitrary element in $C_{2} \tilde{X}^{2}$ is no longer a vector with three $\mathbb{Z} \pi$ entries, but with $3 m n \mathbb{Z}$-entries. Thus, $\partial_{2}$ maps $3 m n$ copies of $\mathbb{Z}$ to $2 m n$ copies of $\mathbb{Z}$, so $\partial_{2}$ is a $2 m n \times 3 m n$ matrix with entries 0,1 , or -1 .

Attaching two three-cells equivariantly to $\tilde{X}^{2}$ is the same as producing a $\pi$-module map $\partial_{3}$ from $\mathbb{Z} \pi \oplus \mathbb{Z} \pi$ to $\operatorname{Ker}\left(\partial_{2}\right)$. (The image of $\partial_{3}$ must be in $\operatorname{Ker}\left(\partial_{2}\right)$ to produce a chain complex.) Since $\mathbb{Z} \pi \oplus \mathbb{Z} \pi$ is a free $\pi$-module, to produce $\partial_{3}$ it is sufficient to "know" $\operatorname{Ker}\left(\partial_{2}\right)$ in the sense of Section $4 \mathrm{~A}$, because one then simply assigns each of the two $\pi$ module generators to any two arbitrarily selected elements of $\operatorname{Ker}\left(\partial_{2}\right)$. The method for computing the kernel of a map between free abelian groups given by a matrix of integers is well known; one simply performs column manipulations until the kernel is transparent. Here, as we do these column manipulations we also correspondingly manipulate the matrices defining the $x$ and $y$ actions. In the end we have $\operatorname{Ker}\left(\partial_{2}\right)$ as a free abelian group of rank $2 m n-1$ with the new $x$ and $y$ matrices.

\section{C. Computation of Homology $\pi$-modules}

Assume now that a $\partial_{3}$ has been created. The resulting three-complex $\tilde{X}^{3}$ is nilpotent if and only if $H_{2} \tilde{X}^{3}$ and $H_{3} \tilde{X}^{3}$ are nilpotent $\pi$-modules. The latter is easy to check because of this proposition [Lewis 1985]:

Theorem 4.1. A nilpotent $\mathbb{Z}$-free $\pi$-module must be $\pi$-trivial if $\pi$ is finite.

$H_{3} \tilde{X}^{3}=\operatorname{Ker}\left(\partial_{3}\right)$ is obviously $\mathbb{Z}$-free, so we need only compute it (as a kernel) and then check that $x=1$ and $y=1$.

The computation of $H_{2} \tilde{X}^{3}$ is the crux of the problem. However, it is important to distinguish the actual computation of this $\pi$-module in the sense of Section 4A from merely determining that $H_{2} \tilde{X}^{3}$ is nilpotent. In either case, one begins the same way. We have $H_{2} \tilde{X}^{3}=\operatorname{Ker}\left(\partial_{2}\right) / \operatorname{Im}\left(\partial_{3}\right)$, the cokernel of $\partial_{3}$. The kernel of $\partial_{2}$ has already been computed. We must "normalize" $\partial_{3}$, that is, put 
$\partial_{3}$ into a form that reveals $\operatorname{Im}\left(\partial_{3}\right)$. The algorithm is similar to the one that computes the kernel of a map by operating on the row space of a matrix via column reductions. This time we operate on the column space of the matrix via row reductions. Each row operation is also performed on the $x$ and $y$ matrices that define the $\pi$-action. The result is an upper-triangular matrix in which each entry is an integer, any zeros on the diagonal are together at the lower end, and each entry in the column above such a zero is zero. $R$ zeros mean that the cokernel $\mathrm{H}_{2} \tilde{X}^{3}$ contains a free abelian group of rank $R$. Experimentally, we find that almost always $R=0$, so we shall assume that here to simplify the exposition. (We found experimentally that, when nilpotent, $H_{3} \tilde{X}^{3}$ is almost always simply $\mathbb{Z}$ with trivial $\pi$-action. Then the Euler characteristic results force $R=0$ for nilpotency.) We could continue manipulating the matrix until it is in Smith normal form, diagonal with each integer entry dividing the next. We do not have to do that to determine nilpotency. We must check that $(x-1)^{k}=0$ for some $k$. This condition holds if and only if every column vector of $(x-1)^{k}$ is in the image of the map defined by the normalized $\partial_{3}$. This amounts to solving a system of linear equations that is upper triangular and therefore easy to program. Similarly for $(y-1)^{k}$. It is not hard to get an upper bound for $k$, based on the product of the entries on the diagonal of the normalized $\partial_{3}$.

Now, should it be desired to actually compute $\mathrm{H}_{2} \tilde{X}^{3}$ in the sense of Section $4 \mathrm{~A}$, the normalizing process must be continued until the Smith Normal Form is attained. If the result is a diagonal matrix of, say, $k$ integers $n_{1}, n_{2}, \ldots, n_{k}$, each dividing the next, then

$$
H_{2} \tilde{X}^{3}=\mathbb{Z}_{n_{1}} \oplus \mathbb{Z}_{n_{2}} \oplus \cdots \oplus \mathbb{Z}_{n_{k}}
$$

as an abelian group, and the new $x$ and $y$ matrices provide the $\pi$-module structure.

\section{THE PROGRAMS}

Programs implementing the ideas of the previous section were written by the student participants (except for the Smith normal form routine, which is a built-in part of Fermat [Lewis 1996]). The programs were initially written in Pascal, following the algorithms described above. Only one point has not been discussed: where do we get the cell attaching map $\partial_{3}$ ? The answer is that we simply create $\partial_{3}$ at random, then check to see that the resulting complex is nilpotent. Recall that to produce $\partial_{3}$ it is sufficient to assign each of the two $\pi$ module generators to any two arbitrarily selected elements of $\operatorname{Ker}\left(\partial_{2}\right)$. The rest of the $2 m n-1 \times 2 m n$ matrix $\partial_{3}$ is filled in by the $x$ and $y$ actions on the two random columns.

In running the program, we found that normalizing this randomly produced matrix $\partial_{3}$ inevitably produced very large integers, beyond the reach of Pascal or any compiled programming language. We tried using well-known computer algebra systems, but were disappointed by the speed. Fermat was found to be enormously faster and more space efficient. However, we needed even more speed, since all complexes for $\pi=\mathbb{Z}_{2} \oplus \mathbb{Z}_{6}$ failed to be nilpotent at the $\mathrm{H}_{2} \tilde{X}^{3}$ stage.

The problem was solved by reducing $\partial_{3}$ modulo $p$ for primes $p$, or, put another way, tensoring all of the chain complexes with $\mathbb{Z}_{p}$. If the complex is nilpotent, the tensored homology modules will be nilpotent for any $p$. By tensoring with the first 42 primes, the program (in Pascal) quickly rejects almost all candidates. The remaining candidates were checked completely by the program written in Fermat. We found many new examples this way of nilpotent complexes with fundamental group $\pi=$ $\mathbb{Z}_{2} \oplus \mathbb{Z}_{2}, \mathbb{Z}_{2} \oplus \mathbb{Z}_{3}, \mathbb{Z}_{2} \oplus \mathbb{Z}_{4}$ and $\mathbb{Z}_{3} \oplus \mathbb{Z}_{3}$. However, these groups are all cyclic or $p$-groups! Our main motivating case, $\mathbb{Z}_{2} \oplus \mathbb{Z}_{6}$, remained elusive: despite hundreds of hours of computer time and several R.E.U. projects, all candidates found by the Pascal phase were rejected by the second Fermat phase: each $H_{2} \tilde{X}^{3}$ contained a perfect submodule whose 
order was a product of primes larger than those on the list of 42 .

Moore provided the idea that finally produced examples over $\mathbb{Z}_{2} \oplus \mathbb{Z}_{6}, \mathbb{Z}_{3} \oplus \mathbb{Z}_{6}$, and $\mathbb{Z}_{2} \oplus \mathbb{Z}_{10}$ : be less random in the assignment of the two random columns of $\partial_{3}$. By placing only 0 and \pm 1 in a sparse enough fashion, success was attained. Some of the nilpotent spaces discovered are listed in Table 1.

\begin{tabular}{|ccc|}
\hline$\pi_{1} X$ & $\operatorname{dim} \partial_{3}$ & $H_{2} \tilde{X}^{3}$ \\
\hline $\mathbb{Z}_{2} \oplus \mathbb{Z}_{6}$ & $23 \times 24$ & $\mathbb{Z}_{2} \oplus \mathbb{Z}_{8}$ \\
& & $\mathbb{Z}_{2} \oplus \mathbb{Z}_{16}$ \\
& & $\mathbb{Z}_{4} \oplus \mathbb{Z}_{8}$ \\
& $\mathbb{Z}_{4} \oplus \mathbb{Z}_{16}$ \\
& & $\mathbb{Z}_{2} \oplus \mathbb{Z}_{2} \oplus \mathbb{Z}_{2}$ \\
& & $\mathbb{Z}_{2} \oplus \mathbb{Z}_{4} \oplus \mathbb{Z}_{8}$ \\
& $\mathbb{Z}_{4} \oplus \mathbb{Z}_{4882576(=16 \cdot 401 \cdot 761)}$ \\
\hline $\mathbb{Z}_{3} \oplus \mathbb{Z}_{6}$ & $35 \times 36$ & $\mathbb{Z}_{3} \oplus \mathbb{Z}_{3} \oplus \mathbb{Z}_{3} \oplus \mathbb{Z}_{18}$ \\
& & $\mathbb{Z}_{3} \oplus \mathbb{Z}_{9} \oplus \mathbb{Z}_{9} \oplus \mathbb{Z}_{9}$ \\
& & $\mathbb{Z}_{3} \oplus \mathbb{Z}_{9} \oplus \mathbb{Z}_{9} \oplus \mathbb{Z}_{18}$ \\
& $\mathbb{Z}_{3} \oplus \mathbb{Z}_{3} \oplus \mathbb{Z}_{3} \oplus \mathbb{Z}_{27}$ \\
\hline $\mathbb{Z}_{2} \oplus \mathbb{Z}_{10}$ & $39 \times 40$ & $\mathbb{Z}_{2} \oplus \mathbb{Z}_{16}$ \\
\hline
\end{tabular}

TABLE 1. Examples of nilpotent spaces discovered. In every case, $H_{3} \tilde{X}^{3}$ is $\mathbb{Z}$ with trivial $\pi$-action.

The programs are explained in greater detail in [Snyder 1989] and [Xu 1992].

\section{AN ALTERNATE APPROACH AND FUTURE WORK}

Recall that the second part of our strategy has been to represent everything as integers. Accordingly, $\partial_{2}$ is a $2 m n \times 3 m n$ matrix of integers. It might be faster computationally and more illuminating theoretically to retain $\partial_{2}$ as a $2 \times 3$ matrix of elements of $\mathbb{Z}[x, y] /\left\langle x^{n}-1, y^{m}-1\right\rangle$, or perhaps just $\mathbb{Z}[x, y]$. A graduate student at Fordham tried this approach but found algorithms difficult to formulate, although some theoretical results were obtained [Xu 1992].

Some future research topics are:

- Compile and catalog the hundreds of new examples of nilpotent spaces.
- Search for homotopy equivalences among the examples.

- Continue the strategy of working over $\mathbb{Z} \pi=$ $\mathbb{Z}[x, y] /\left\langle x^{n}-1, y^{m}-1\right\rangle$.

- Extend to more general nilpotent fundamental groups.

\section{ELECTRONIC AVAILABILITY}

The programs described in this article are available by request from Lewis. Fermat is available at http://www.bway.net/ lewis.

\section{REFERENCES}

[Brown and Kahn 1977] K. S. Brown and P. J. Kahn, "Homotopy dimension and simple cohomological dimension of spaces", Comment. Math. Helv. 52:1 (1977), 111-127.

[Dror 1971] E. Dror, "A generalization of the Whitehead theorem", pp. 13-22 in Symposium on Algebraic Topology (Battelle Center, Seattle, 1971), edited by P. J. Hilton, Lecture Notes in Math. 249, Springer, Berlin, 1971.

[Lewis 1985] R. H. Lewis, "Homology and cell structure of nilpotent spaces", Trans. Amer. Math. Soc. 290:2 (1985), 747-760.

[Lewis 1996] R. H. Lewis, "Fermat: A computer algebra system for Macs", 1996. See http://www.bway.net/ lewis/.

[Milnor 1956] J. Milnor, "Construction of universal bundles I", Ann. of Math. (2) 63 (1956), 272-284.

[Rotman 1988] J. J. Rotman, An introduction to algebraic topology, Graduate Texts in Mathematics, Springer-Verlag, New York, 1988.

[Snyder 1989] C. M. Snyder, The search for threedimensional nilpotent complexes of finite abelian fundamental group: an algorithm, B.A. thesis, Math. Dept., Fordham University, 1989.

[Spanier 1966] E. H. Spanier, Algebraic topology, McGraw-Hill, New York, 1966.

[Xu 1992] J. Xu, On the construction of finite threedimensional nilpotent spaces, Master's thesis, Math. Dept., Fordham University, 1992. 
246 Experimental Mathematics, Vol. 6 (1997), No. 3

Robert H. Lewis, Department of Mathematics, Fordham University, Bronx NY 10458 (lewis@bway.net)

Guy D. Moore, Princeton University, Joseph Henry Laboratories, PO Box 708, Princeton, NJ 08544

Received March 12, 1996; accepted in revised form April 15, 1997 